\title{
Modeling of passive forces of machine tool covers
}

\author{
Petr KOLAR ${ }^{1}$, Jan HUDEC ${ }^{1}$, Matej SULITKA ${ }^{1}$, Martin LACHMAN ${ }^{2}$ and Radomir MENDRICKY ${ }^{2}$ \\ ${ }^{1}$ Research Center of Manufacturing Technology, Czech Technical University in Prague, Czech Republic \\ ${ }^{2}$ Research Center of Manufacturing Technology, Technical University of Liberec, Czech Republic \\ ${ }^{1}$ p.kolar@rcmt.cvut.cz (corresponding author)
}

\begin{abstract}
:
The passive forces acting against the drive force are phenomenon that influences dynamical properties and precision of linear axes equipped with feed drives. Covers are one of important source of passive forces in machine tools. The paper describes virtual evaluation of cover passive forces using the cover complex model. The model is able to compute interaction between flexible cover segments and sealing wiper. The results are deformation of cover segments and wipers which is used together with measured friction coefficient for computation of cover total passive force. This resulting passive force is dependent on cover position. Comparison of computational results and measurement on the real cover is presented in the paper.
\end{abstract}

Keywords: Machine tools covers, passive force modeling, passive force compensation

\section{Introduction}

Mathematical modeling of machine tool main structures is nowadays in very advanced stage already. That is the reason why manufacturers begin also to focus on other machine tool components, e.g. telescopic covers.

The concept of virtual machine [1] enables us to provide virtual testing of machine tool prototypes. Therefore the machine properties could be directly computed instead of properties prediction from indirectly parameters like statical and dynamical stiffness. Complex modeling of feed drive axes [2], [3] is one of possible tools for prediction of movable axes accuracy. Better results of these models can be reached using results of models of other mechanical components. Especially modeling of covers is important for building of more reliable models of feed axes.

Covers are very important part of machine tool. They have direct influence on machine reliability and components lifetime. The most used design of modern covers is telescopic cover, which consists of sheet metal segments, polyurethane sealing wipers, guiding scissor mechanisms and other components. However, covers are also source of passive forces. Therefore they could influence feed drives and they could have significant impact on machine accuracy, especially on low speed through slip-stick effect. They also increase power demand on drives.

Chapter 2 deals about experimental tests on existing machines. Influence of passive forces on machine tool will be demonstrated and discussed on examples of two different machines.

Chapter 3 deals about complex model of cover. Experiments on test bed described in chapter 4 are used for verification of computed results.

\section{Experimental tests on existing machines}

\subsection{Measurement on vertical milling machine}

There has been done a measurement of passive resistance force of covers on the 3 -axes milling machine. This machine is a vertical milling center with horizontal cross table ( $\mathrm{X}$ and $\mathrm{Y}$ axes) and vertical spindle axis $\mathrm{Z}$. The table axes are covered by 4 covers ( 2 covers per axis).

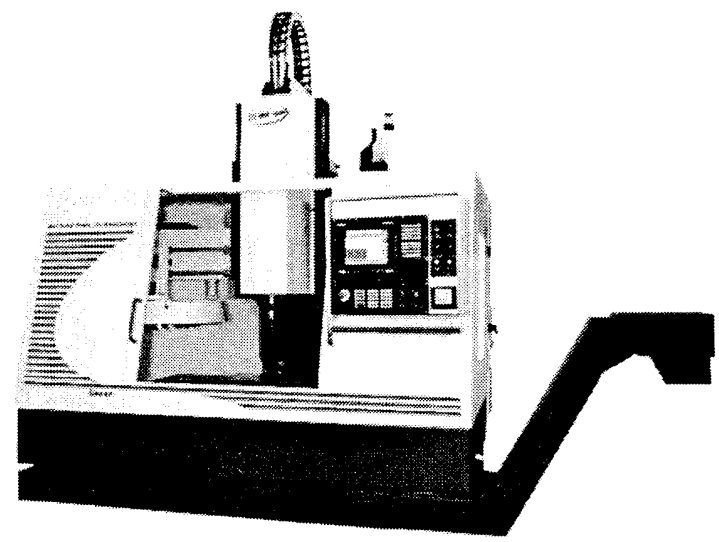

Figure 1: Tested vertical milling centre.

The total resistance force was measured using measurement of motor current. The results are presented on Fig. 2 and Fig. 3. There is no inertial force during continuous motion with constant speed so the measured current shows the force necessary to deal with passive resistance mostly caused by friction.

Subtracting resistance force of axis without covers from force of axis with covers mounted gives the value of force necessary to cope with covers. Is can be seen on following pictures, the cover passive force is responsible for approximately $50 \%$ of whole passive force in this case. 


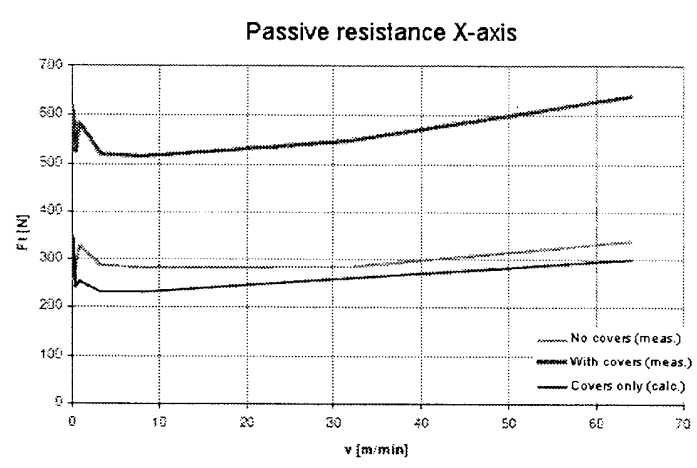

Figure 2: Results of measurement of X-axis.

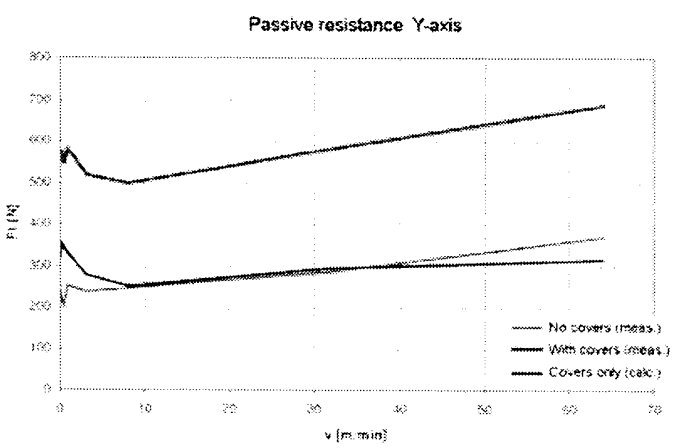

Figure 3: Results of measurement of Y-axis.

The fact that power consumed by cover movement may achieve very high percentage of total power implies the necessity of prediction of passive resistance prior drive design. This prediction can be made by mathematical model in case of development of a new machine.

\subsection{Measurement on horizontal milling machine}

A simulation of circular interpolation has been verified on XY vertical table of horizontal milling center.

This milling center is equipped by two-axis cover that is able to move in two directions simultaneously. That means the cover is moved by lighter $\mathrm{Y}$ axis which is positioned by heavier $\mathrm{X}$ axis.

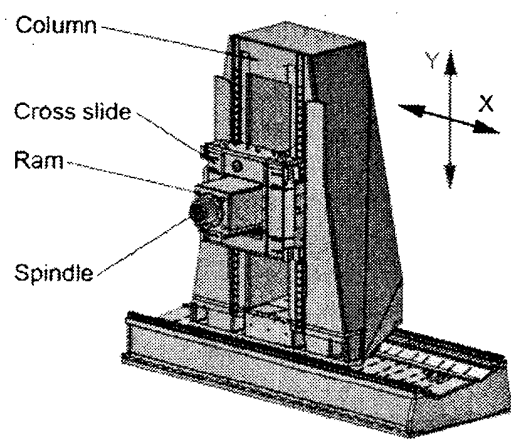

Figure 4: Horizontal milling center.

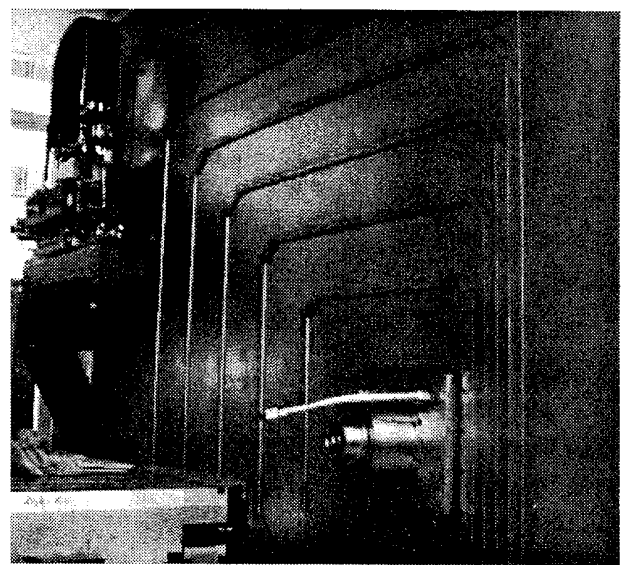

Figure 5: Two-axis cover on horizontal milling machine.

A model with linear force increase has been developed to simulate frictional behavior of axes. This model substitutes the discontinuity between static and dynamic friction by two linear curves. First steep curve represents growth of friction up to value of Coulomb friction. The second curve respects the effect of viscous friction.

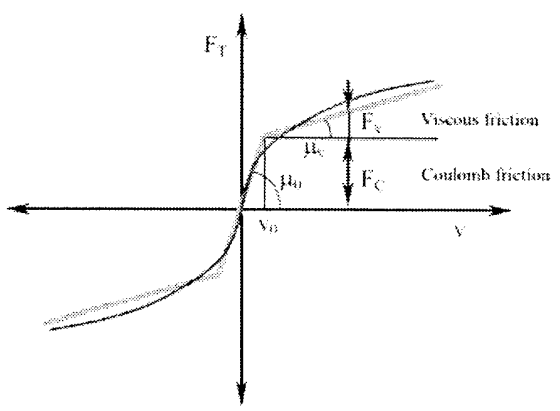

Figure 6: Friction model.

This friction model and model of movable axes [3] have been implemented into simulation model of circular interpolation.

The model parameters have been set up according to previous measurement to allow result comparison.

Friction force is applied to a place of ball nut mounting interface. Simulated circle diameter is $50 \mathrm{~mm}$; velocity is $7 \mathrm{~m} \cdot \mathrm{min}^{-1}$.

Comparison between measured and computed results is shown on Fig. 7. The peaks in diagram show Coulomb friction values. Coulomb friction of X-axis is $4790 \mathrm{~N}$, in case of $\mathrm{Y}$-axis it is $1830 \mathrm{~N}$. We can also see bigger differences between both data sets in $\mathrm{Y}$ axis (vertical axis). The reason is, models of both axes used the same friction model. However, the cover has rectangular shape, therefore both movement directions have different total length of sealing wipers. This is not respected in the friction model, so the $\mathrm{Y}$ axis has worse concordance between simulation and model. It shows need of more accurate modeling of cover passive forces. 


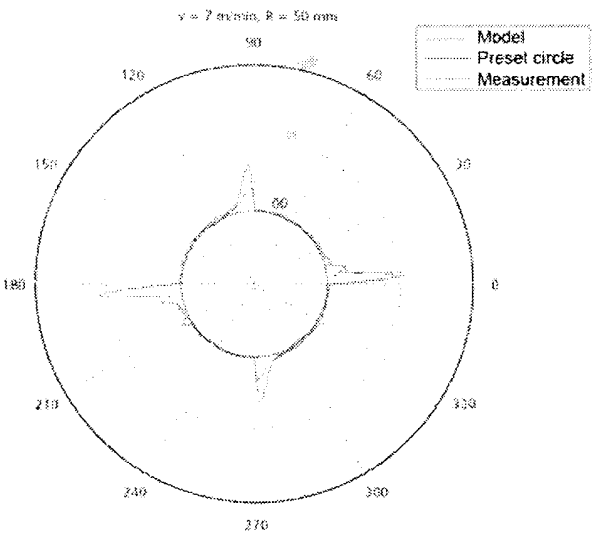

Figure 7: Circular interpolation plot.

\section{Structure of the cover complex model}

In order to compensate friction or / and inertial forces it is necessary to know the passive resistance of the axis and cover. For this and many other reasons a complex model of telescopic cover has been developed (Fig. 8).

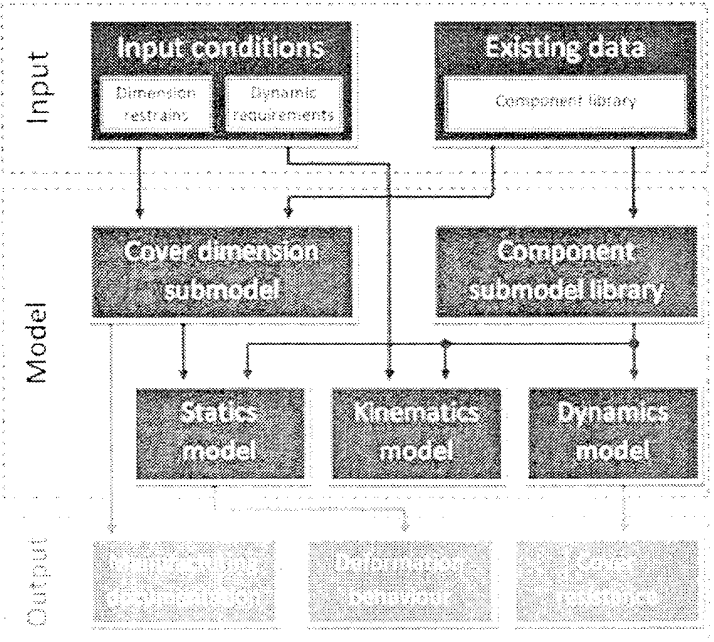

Figure 8: Complex model scheme.

The complex model consists of three basic layers: input data layer, model layer and output data layer [4].

\subsection{Input data}

Input data layer acts as database of model settings. It consists of two main elements - input conditions and existing data.

Input conditions are information containing dimensional and dynamic restrains specified by the customer in the inquiry form. The existing data is a database of cover components - its physical properties and capabilities.

\subsection{Model layer}

The model layer contains mathematical models calculating physical behavior and interactions of parts according to specified input parameters and component properties.

This layer is consists of several models and submodels.

The cover dimension submodel suggests all dimensions of cover optimized for required travel. Optimization is based on dimensional restrains of customer machine tool and dimensional parameters of used components. This assures minimal reasonable proportions for reduction of material consumption, inertial forces and costs.

The component submodel contains mathematical description of components behavior based on its specific properties (mass, stiffness). These characteristics are used in superior interaction models.

Very important submodel is submodel of sealing wiper. The wiper submodel is based on Neo-Hookean hyperelastic constitutive model that is used for wiper mechanical response modelling. A 2D model was created for simulation of wiper interaction between cover segments. Plane strain conditions were assumed. Computational model includes PU wiper, clamping steel profile and interacting adjacent metal sheet. To simulate large deformation effects eight-node element PLANE 183 was used. Wiper interaction with clamping profile and metal sheet was represented by CONTA172/TARGE169 contact element. Example of wiper deformed shape is on Fig. 9. Stiffness characteristic of the wiper is bilinear - see Fig. 10. The model results has been successfully verified with experimental measurement.

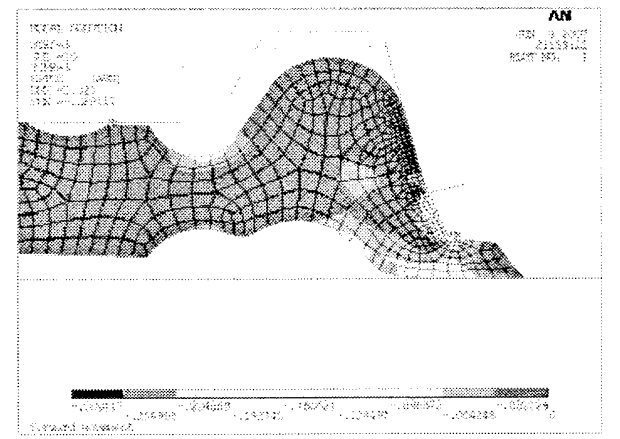

Figure 9: FEM model of deformed wiper

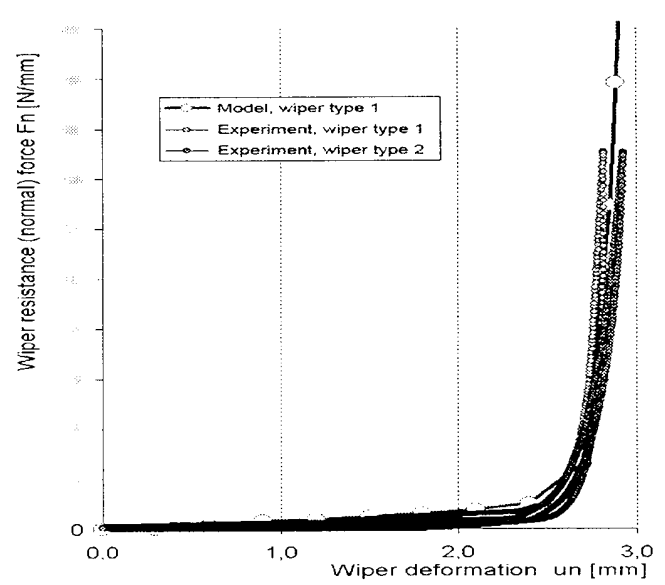

Figure 10: Bilinear stiffness characteristic.

The statics model of cover calculates strain and deformation of particular components. It is possible to predict behavior of operational covers when function and life is concerned. This prevents unacceptable deformations and excessive loads of components. 
The kinematics model describes motion of movable parts and mechanisms dependent on axes movement to reveal necessary space and possible collisions. It is also a data source for dynamics model of covers.

The dynamics model determines actual accelerations and dynamic effect on particular parts of a cover. Along with resistance forces (inertial and friction), these effects represent overall resistance against movement. Resistance is an error value important for axis drive dimensioning.

\subsection{Output data}

Output data layer contains information crucial for actual production and successful implementation of covers into machine tool. This information sets are the basis for automatically generated manufacturing documentation.

It also allows checking the behavior of operational covers - load, wear and deformation of components. That is important for designers to verify the sufficiency of their solution (Fig. 14).

The last output is overall cover resistance. This data are important for determination of drive axis load caused by covers. That is the very important information for customer.

Cover is usually one of the last parts mounted to the machine because it is requested when all parts of the machine (including drives) are specified for order or designed and ready for production.

Problem could happen if the cover was not taken into account during drive dimensioning. There is a significant difference in machine drive dynamics when cover is mounted to the machine. Drive positioning is less responsive and power consumption is $10-30 \%$ higher in case of two axis covers.

Producer is usually unable to provide such an information, definitely not before the cover is produced. This model can estimate cover resistance during the price offer process before the cover is designed. These are parameters important for compensation.

That is the added value of the complex model.

\subsection{Passive resistance by model}

One of the complex model output is calculation of cover deflection used for estimation of normal force generated by wiper preload. This force can be transformed into friction force of wipers.

\section{Verification}

Results of friction force calculated by the complex model have been verified by an experiment.

\subsection{Tests description}

Tested telescopic one-axis cover is mounted on a testing bed. The cover is supported by sliding rails and its left side is actuated by movable table driven by ball screw with motor. Motor power is $2 \mathrm{~kW}$ and torque is $10 \mathrm{Nm}$, ball screw diameter is $32 \mathrm{~mm}$, pitch $32 \mathrm{~mm}$.

The feed drive axis is controlled in velocity loop and the value of current is recorded for further analysis.

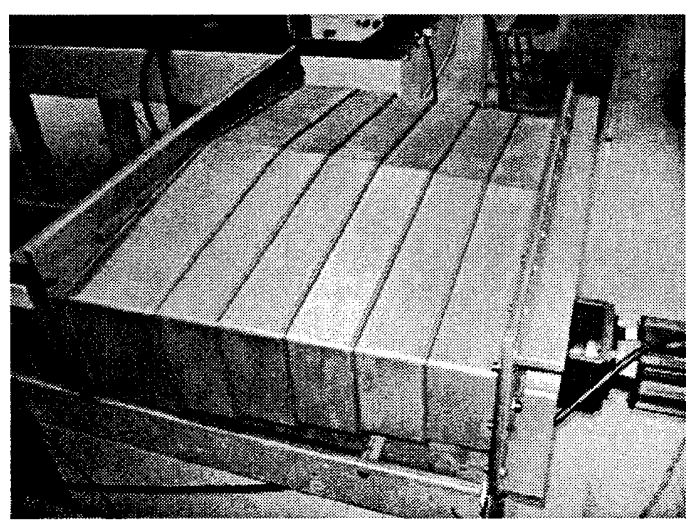

Figure 11: The testing bed setup with cover.

\subsection{Tests results}

The testing cycle has been set to extend the cover by $400 \mathrm{~mm}$, remain stopped for $0.5 \mathrm{sec}$, return, stop and repeat the cycle. The extension / contraction velocity was $30 \mathrm{~m} \cdot \mathrm{min}^{-1}$.

The recorded data of current values have been processed to plot corresponding force behavior. The figure 11 shows the force behavior with and without the cover mounted.

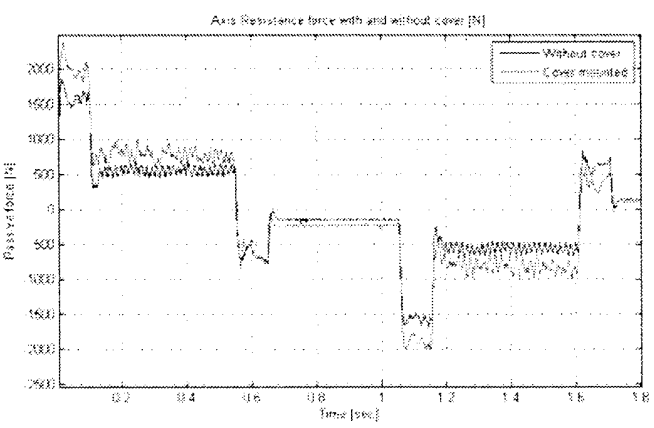

Figure 12: Resistance force of the whole axis with (green) and without cover (blue).

Subtraction of these two characteristics gives behavior of the telescopic cover. Peaks show inertial forces during acceleration and stable parts of curves show value of the passive resistance during motion. Following figure compares simulated and measured passive resistance of covers. 


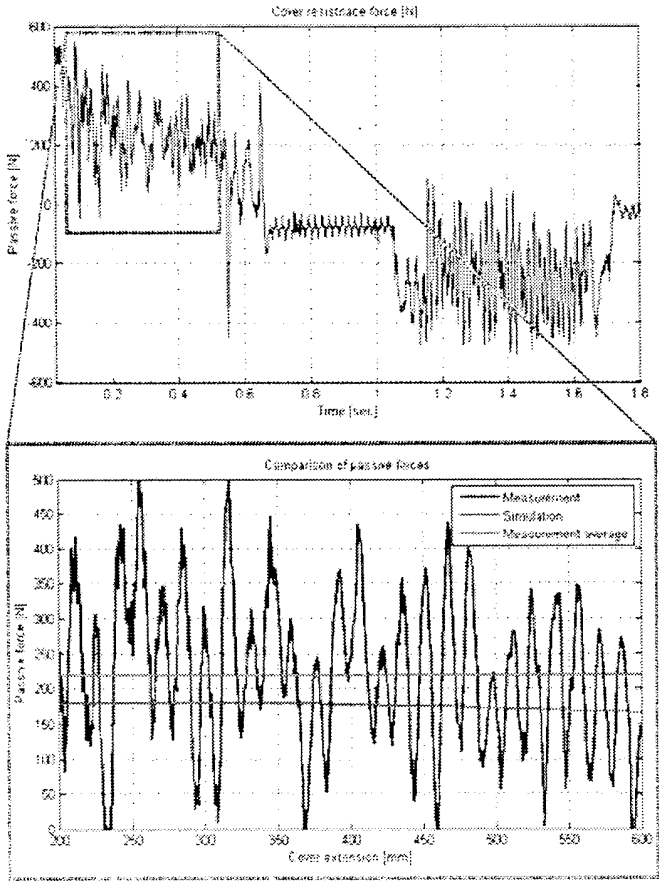

Figure 13: Comparison of measured and simulated passive resistance forces during cover extension.

\subsection{Results discussion}

The simulated values however contain friction caused by wiper so far. The average value of simulated friction force is approx. $170 \mathrm{~N}$.

The measured values contain overall passive resistance of the whole cover including sealing wiper and guiding parts. This measured values show dynamic friction. The average value of measured friction force is approx. $220 \mathrm{~N}$.

The difference between model and measurement is caused by absence of simulation of guiding sliders and other factors described below.

The high frequency fluctuation of measured force is caused by fluctuation of current of the motor during its rotation due to pole pitch.

The low frequency fluctuation of measured force was not unfortunately identified. Because this fluctuation occurs also during movement without covers, it is probably problem of the test bed design.

According to the model, the friction force slightly weakens (fig 13). This phenomenon respects higher rigidity of the cover when contracted, because wipers are located above each other supporting each other. In this situation the metal segment cannot be bended by the wiper preload like it is when the cover is extended. When the preload is not released by segment deformation it applies more force to the cover generating more friction (Fig. 14).

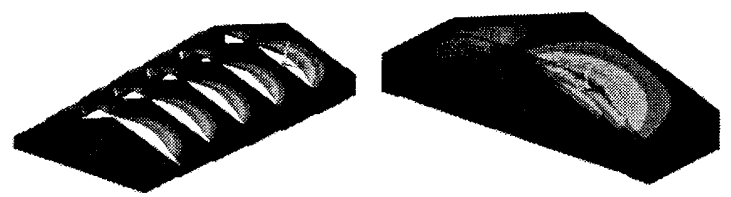

Figure 14: Deformatrions on extended and contracted cover.

\subsection{Factors affecting model accuracy}

Unfortunately there are plenty of factors having substantial influence to result of the simulated value of passive resistance.

One unpredictable factor is the assembly process. The best assembly tolerance of wiper preload is $+/-0.2 \mathrm{~mm}$. This can affect the result by $5-50 \%$ [5].

Under ideal conditions the friction is also dependent on its current extension of the cover as mentioned in previous chapter; and it is dependent on the current direction of movement as the sealing wiper produces more friction in one direction than in the other. These factors seem insignificant in real situation.

Current version of complex model estimate static friction only. Real friction during movement is dynamic and is speed-dependent.

Other factors are temperature, age of material, friction contact conditions (oil, cutting fluid, dry friction).

\subsection{Factors affecting axis dynamics related to covers}

Inertial forces of cover are responsible for significant reduction of drive parameters during acceleration and deceleration of drive.

Frictional resistance and its changes is another factor limiting drive positioning precision. This paper describes methods of frictional resistance prediction in order to give information for its compensation.

Ratio of axis and cover weights is also very important. There is very often problem on cross table structures: one drive is weaker, because it accelerates just a table. Another drive is stronger, because it accelerate the whole cross slide and the table. But covers are similar on both axes, therefore the lighter axis positioning results are more afflicted.

\section{Conclusion}

This paper describes current stage of passive resistance model of covers. This model is a part of virtual machine concept.

Results of passive force measurement of machine axes and covers are presented as well as methods of their prediction. The differences between computed and measured average values are influenced with missing of description of sliding elements passive forces. It will be a future work.

The predicted value of cover passive forces can be used for more precise choice of drives during development process. The model is also able to predict caver passive forces in dependence on cover position and velocity. This data could be used as feedforward signal for quadrant error compensation. This application will be also next work. 


\section{Acknowledgement}

This research has been supported by the 1M0507 grant of the Ministry of Education of the Czech Republic.

\section{References}

[1] Altintas, Y., Brecher, C., Weck, M., Witt, S., Virtual Machine Tool. Annals of CIRP Vol. 54, Issue 2, 2005 p. $115-138$.

[2] Sulitka, M. et all, Machine Tool Lightweight Design and Advanced Control Techniques. International Congress MATAR PRAHA 2008 - Proceedings of Part 1: Drives \& Control, Design, Models \& Simulation. Prague, Society for machine tools, 2008, p. 129 - 134. ISBN 978-80-903421-9-4.
[3] Vesely, J., Sulitka, M., Machine Tool Virtual Model. International Congress MATAR PRAHA 2008 Proceedings of Part 1: Drives \& Control, Design, Models \& Simulation. Prague, Society for machine tools, 2008, p. 115 - 122. ISBN 978-80-903421-9-4.

[4] Hudec, J., Kolar, P., "Complex Model Of Two-axis Covers: Introduction and Sealing Wiper Behaviour", in: Manufacturing Intelligent Design and Optimization, Journal of Machine Engineering Vol. 7, No.1, Wroclaw 2007, ISSN 1895-7595

[5] Hudec, J., Skacel, P., Kolar, P., Prediction of Sealing Wiper Resistance Force. 8th Youth Symposium on Experimental Solid Mechanics, Györ 2009 [poster] 